\title{
On the Thermal Stability of [60]fullerene
}

\section{cycloadducts: Retro-Cycloaddition Reaction of}

\section{2-Pyrazolino[4,5:1,2][60] fullerene.}

Juan Luis Delgado, ${ }^{\dagger}$ Frédéric Oswald, ${ }^{\dagger}$ François Cardinali, ${ }^{\ddagger}$ Fernando Langa, ${ }^{* \dagger}$ and Nazario Martín ${ }^{* \dagger \$}$

Departamento de Química Orgánica, Facultad de Ciencias Químicas, Universidad Complutense de Madrid, 28040, Madrid, Spain

Instituto de Nanociencia, Nanotecnología y Materiales Moleculares (INAMOL), Universidad de Castilla-La Mancha, 45071 Toledo, Spain

IMDEA-Nanociencia, Facultad de Ciencias, Módulo C-IX, $3^{a}$ planta, Ciudad Universitaria de Cantoblanco, 28049 Madrid.

nazmar@quim.ucm.es; Fernando.LPuente@uclm.es 


\section{CONTENTS OF SUPPORTING INFORMATION}

General procedure for the retrocycloaddition experiments..............................................S3

Synthesis and characterization of the precursor hydrazone $(\mathbf{h}-\mathbf{2 d})$, characterization of $\mathbf{2 d}$.....S3

Characterization of $\mathbf{2 f}$...

Typical profile for the microwave reactions.

S10 


\section{General procedure for the retrocycloaddition experiments:}

All the reactions were monitored by HPLC (Cosmosil Buckyprep column, $4.6 \mathrm{~mm}$ (i.d.) F $250 \mathrm{~mm}$; toluene, flow rate: $1 \mathrm{mLmin}^{-1}$ ). Retention times: $7.2-13.5 \mathrm{~min}$ for the 2-pyrazolinofullerene; $8.0 \mathrm{~min}$ for $\mathrm{C}_{60}$.

\section{Experiments o-DCB-24h and $48 \mathrm{~h}$.}

In a typical experiment, 2-pyrazolinofullerene $(0.005 \mathrm{~mol})$,) were heated at reflux in $o$-DCB $(5 \mathrm{~mL})$.

\section{Experiments MA-24h. and $48 \mathrm{~h}$.}

2-pyrazolinofullerene $(0.005 \mathrm{~mol})$ and maleic anhydride $(0.016 \mathrm{mmol})$, were heated at reflux in $o$-DCB (5 mL).

Experiments CuTf 24 h. and 48 h.

2-pyrazolinofullerene $(0.005 \mathrm{~mol})$ and copper (II) triflate $(0.005 \mathrm{mmol})$ were heated at reflux in $o$-DCB $(5 \mathrm{~mL})$.

\section{Experiments MA-CuTf ${ }_{2}-24 h$. and 48 h.}

2-pyrazolinofullerene $(0.005 \mathrm{~mol})$, maleic anhydride $(0.016 \mathrm{mmol})$, and copper (II) triflate $(0.005$ mmol) were heated at reflux in $o$-DCB $(5 \mathrm{~mL})$.

\section{Synthesis and characterization of}

(E)-1-(4-methoxyphenyl)-2-(4-nitrobenzylidene)hydrazone (h-2d)

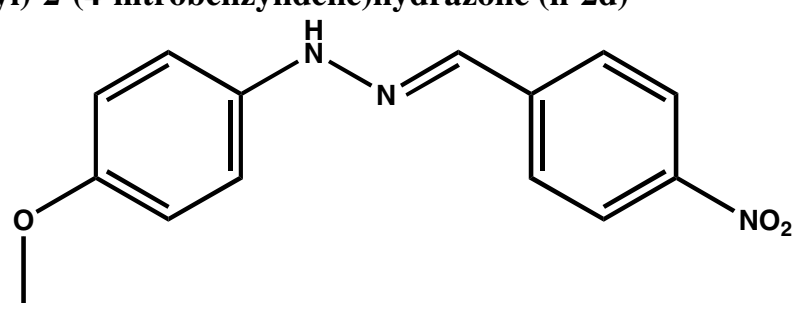

An ethanol solution $(30 \mathrm{~mL})$ of 4-nitrobenzaldehyde $(236 \mathrm{mg}, \quad 1.56 \mathrm{mmol})$ and 4methoxyphenylhydrazine hydrochloride $(274 \mathrm{mg}, 1.56 \mathrm{mmol})$ with 2 drops of acetic acid was refluxed during $2 \mathrm{~h}$, then evaporated. The residue was recrystallized two times by dissolution in the minimum hot chloroform which was then poured in cold pentane. Red crystals precipitated that were isolated by filtration.

$237 \mathrm{mg}(56 \%)$ were obtained. m.p. $165^{\circ} \mathrm{C} .{ }^{1} \mathrm{H}$ NMR (400 MHz, $\left.\mathrm{CDCl}_{3}\right) \delta 8.22$ (d, J=8.0 Hz, 2H), 7.67 (s, 1H), 7.5 (d, J=8.0 Hz, 2H), 7.11 (d, J=7.2 Hz, 2H), 6.90 (d, J=8.4 Hz, 2H), 3.81 (s, 3H). ${ }^{13} \mathrm{C}$ NMR (100 $\left.\mathrm{MHz}, \mathrm{CDCl}_{3}\right) \delta 126.0,124.1,114.8,114.4$, 55.7. FT-IR (KBr): 3285, 1595, 1561, 1528, 1506, 1499, $1467,1446,1410,1335,1261,1232,1172,1148,1105,1096,1023,908,856,847,820,762,748,692$, $632,617 \mathrm{~cm}^{-1}$. UV-Vis $\lambda$ (ع) 430 (18700), 273 (20300). 
Nuclear Magnetic Resonance

(h-2d) Hydrazone ${ }^{1}$ H-NMR. ${ }^{13} \mathrm{C}-\mathrm{NMR}$
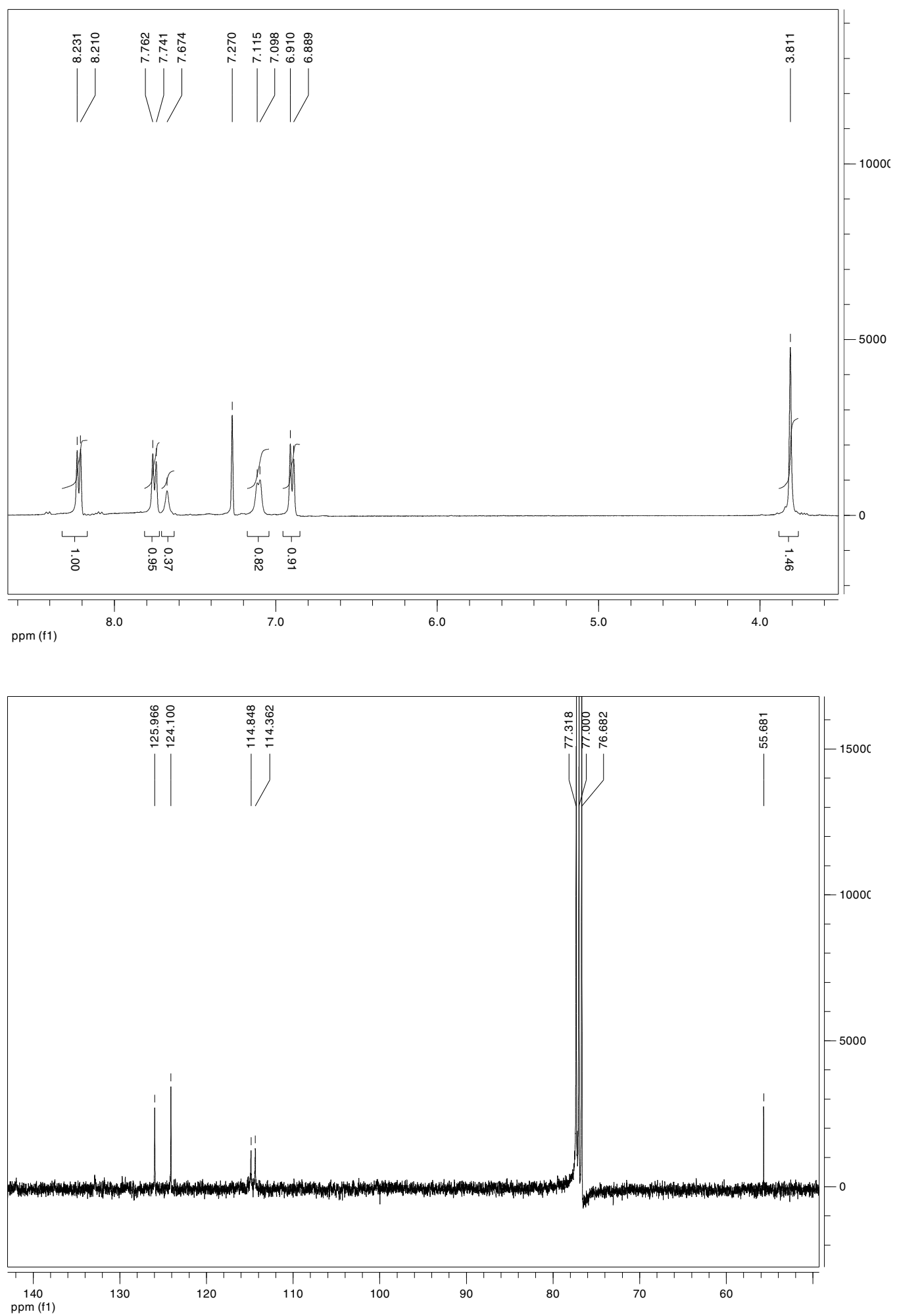
2d ${ }^{1} \mathrm{H}$-NMR and ${ }^{13} \mathrm{C}-\mathrm{NMR}$

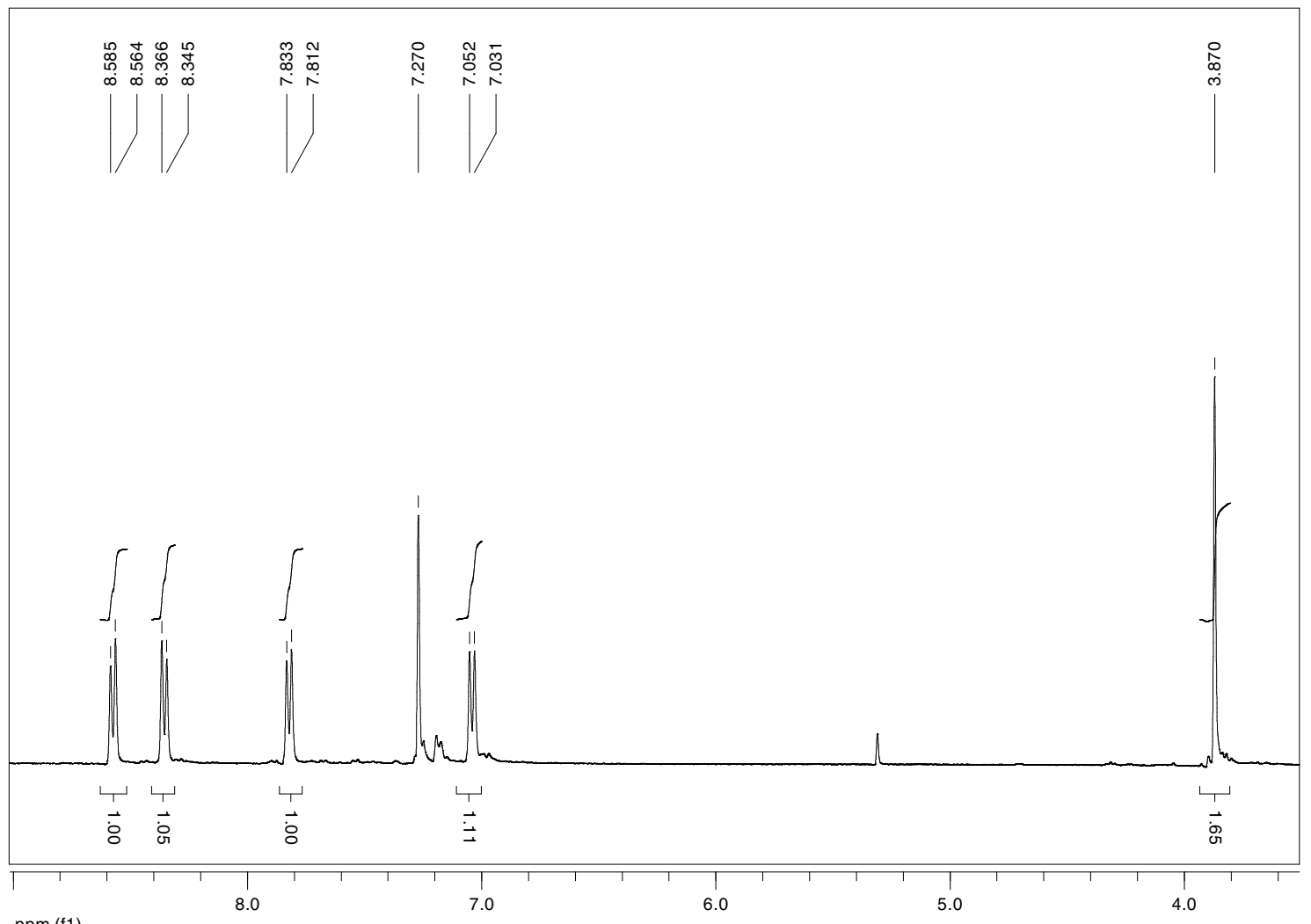

ppm (f1)

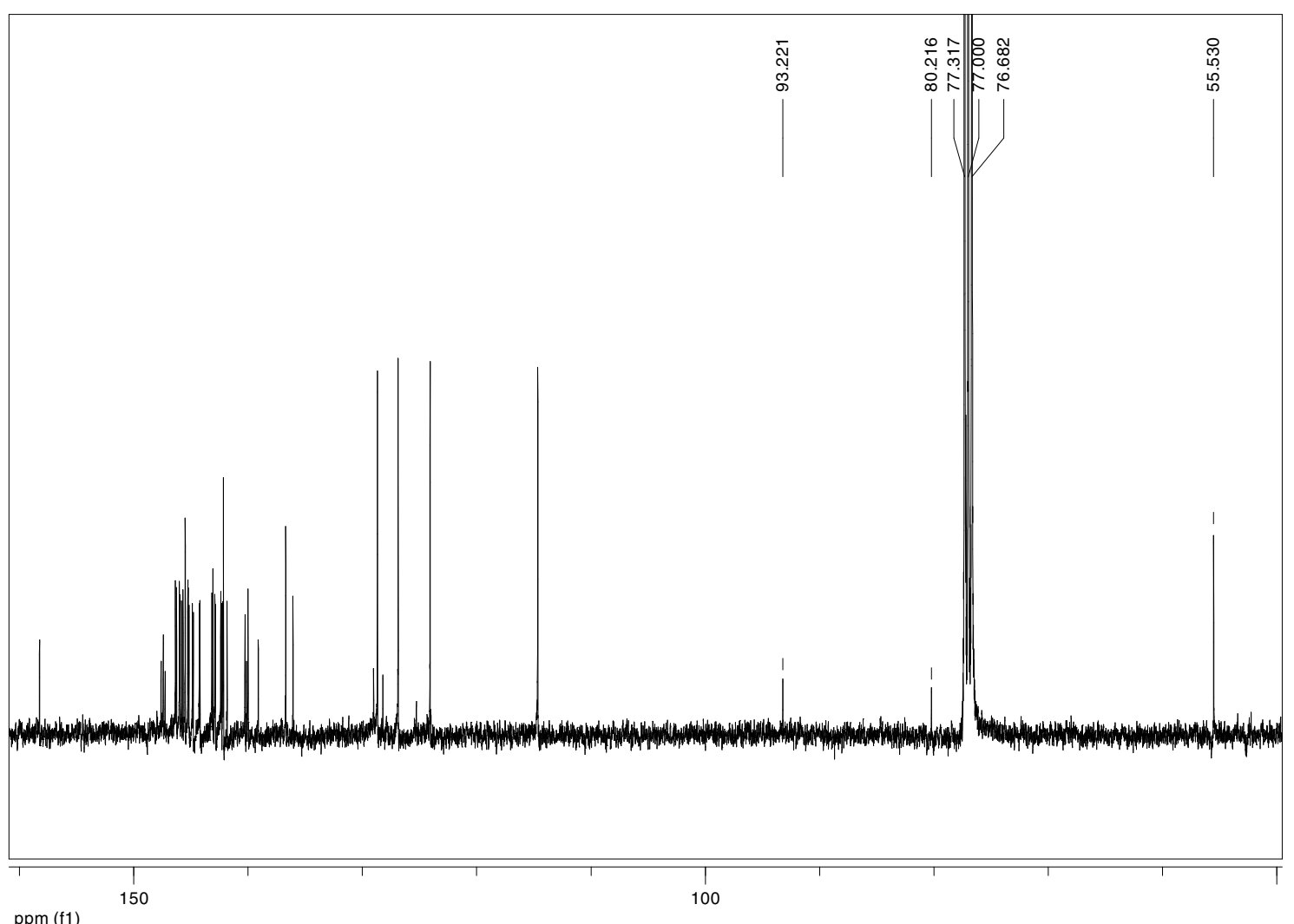




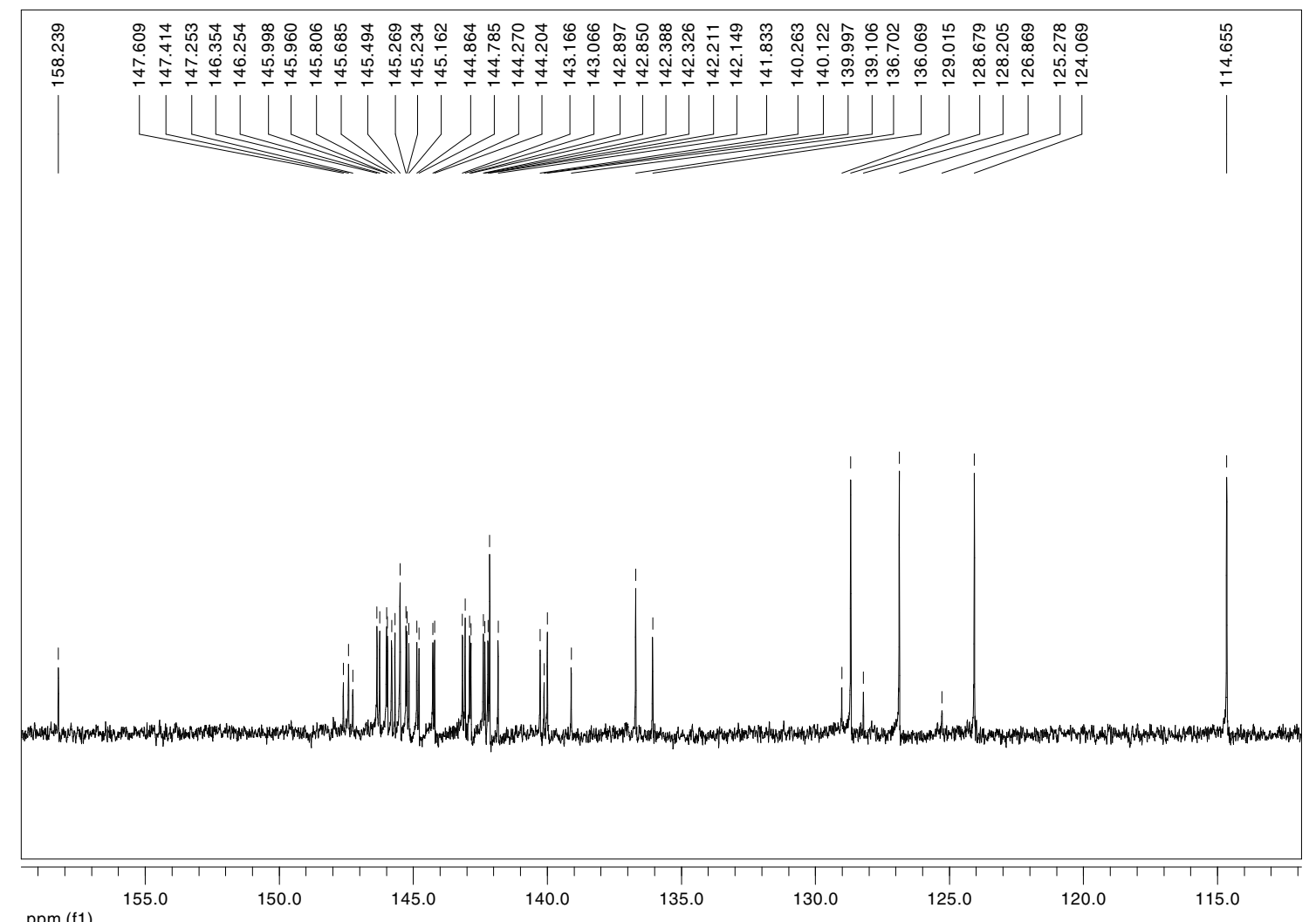

MS 2d

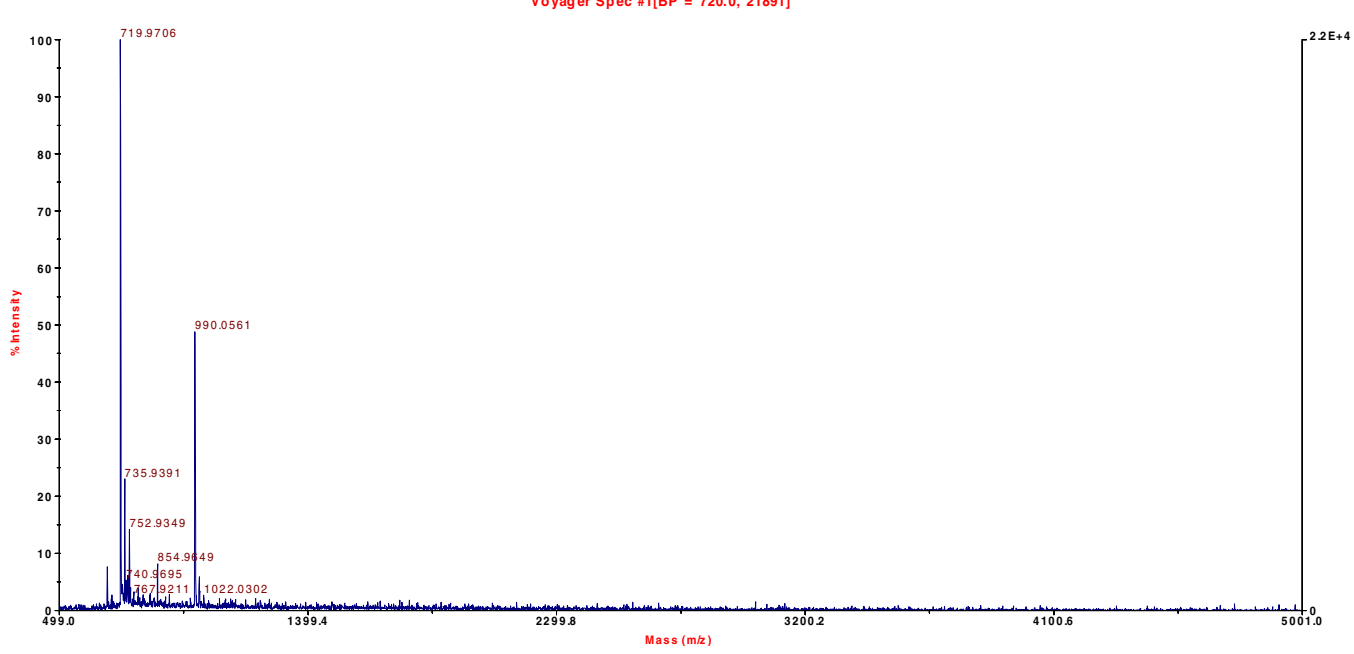


MS 2d

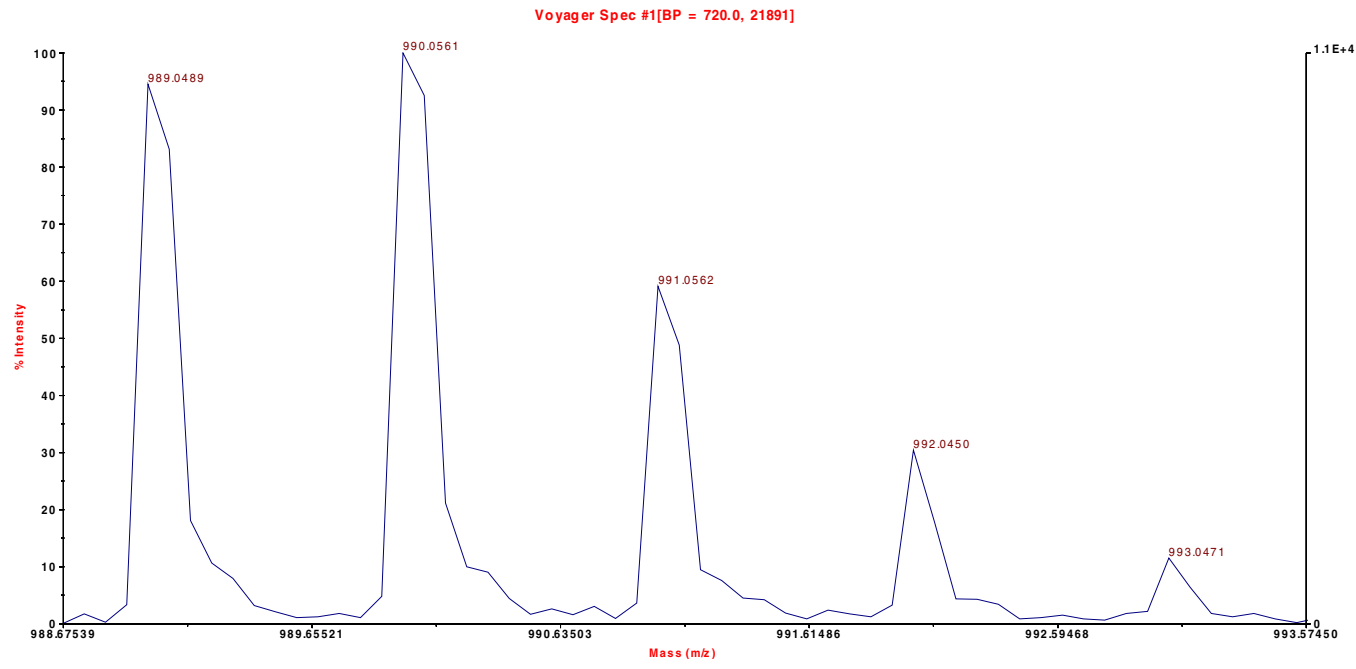

\section{HPLC}

2d: in toluene $1 \mathrm{ml} / \mathrm{min}$

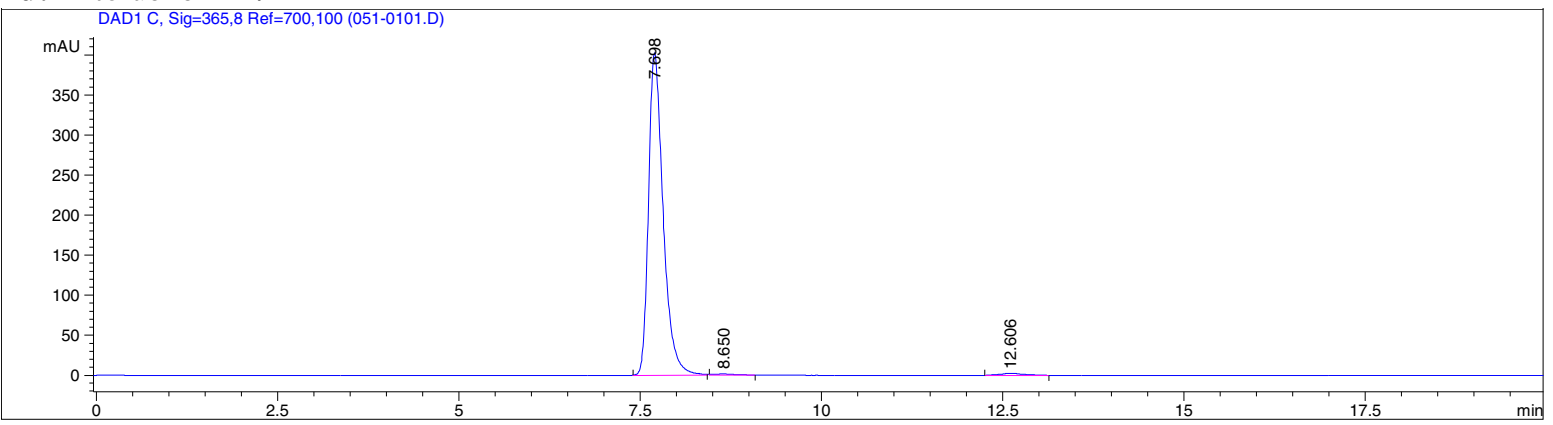




\section{Characterization of $2 f$}

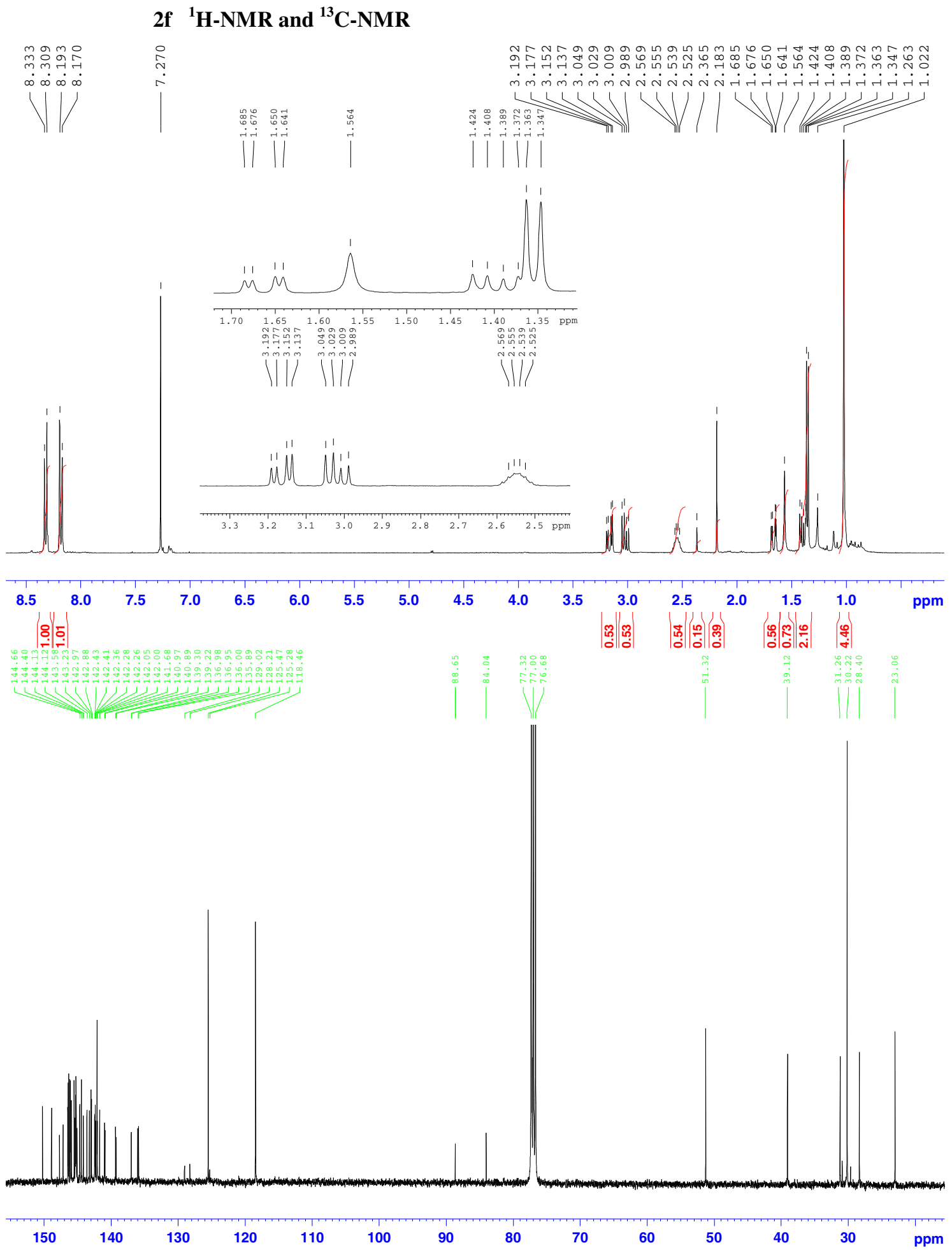


MS-MALDI 2e

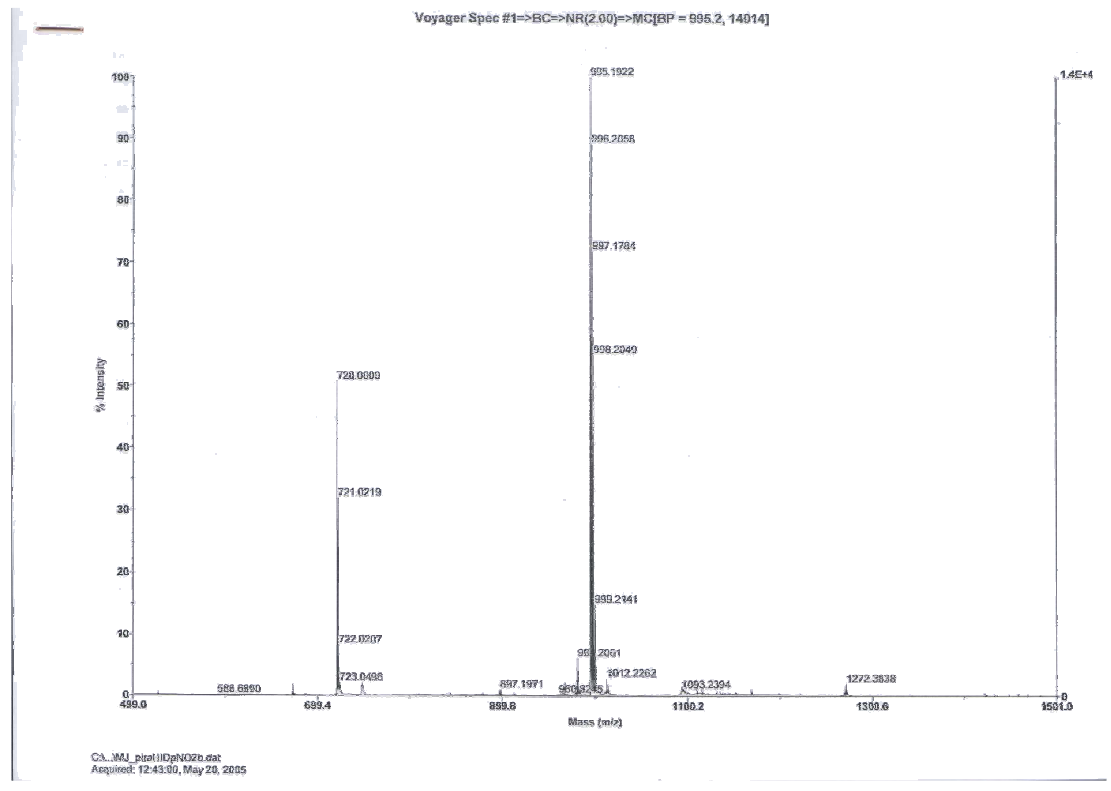

HPLC

2f: in toluene/hexane (7:3) $1 \mathrm{ml} / \mathrm{min}$

Data Eile D: \DATOS RUBEN \RC-2-143.

Sample Name: Retro
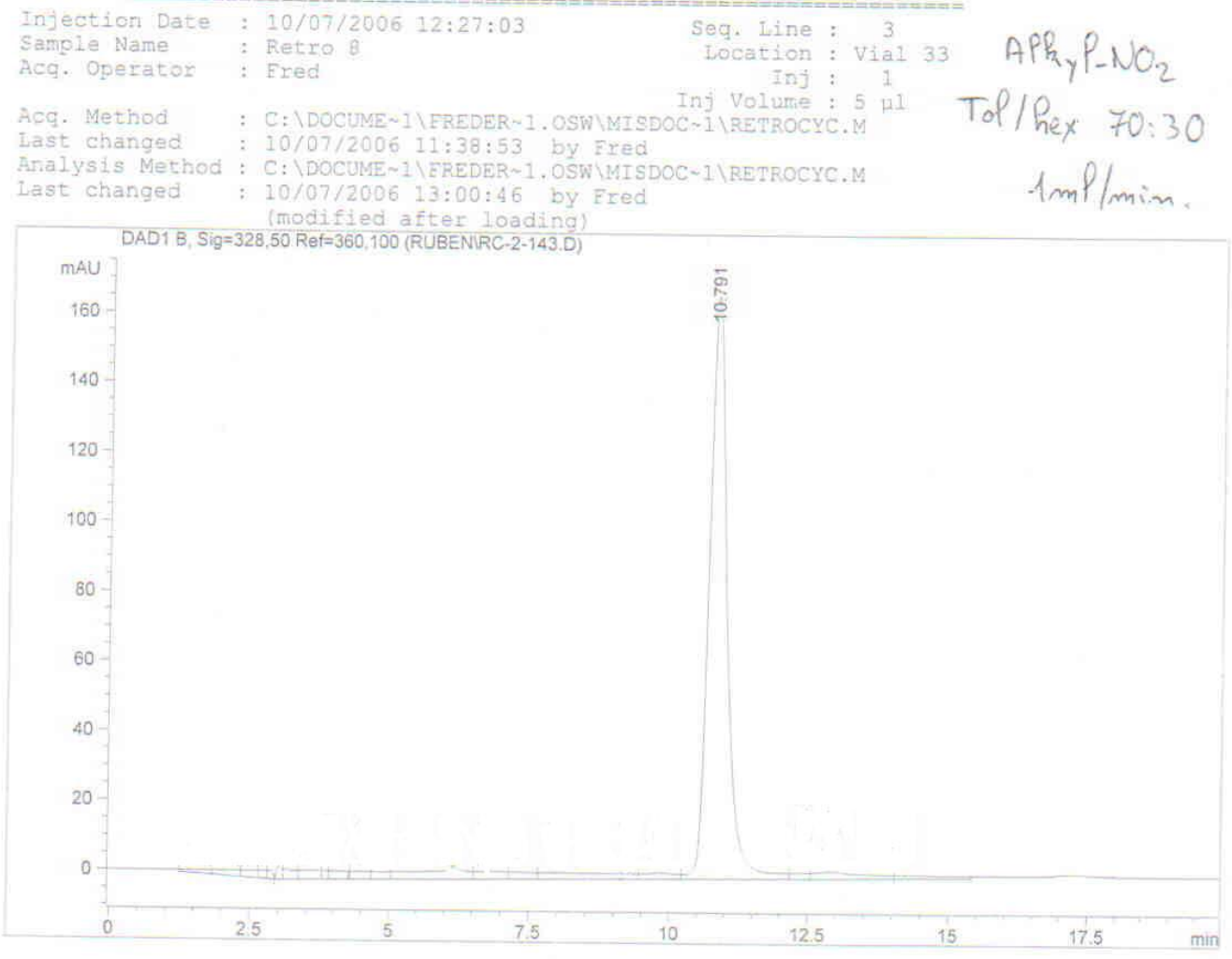
Typical profile for the microwave reactions (4hours each time):

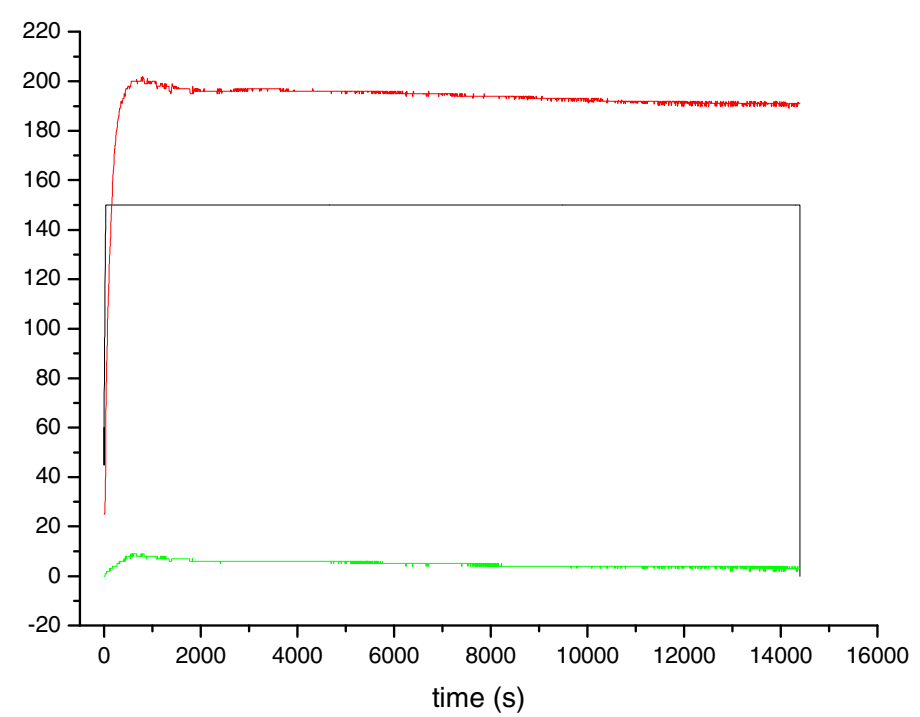

Figure 1: temperature (red), power (black), pressure (green). 